\title{
Ero sempre una betulla che si aggirava in un boschetto di pioppi
}

Jarmila Očkayová

\section{(2) OpenEdition}

12 Journals

Edizione digitale

URL: https://journals.openedition.org/cher/11116

DOI: 10.4000/cher.11116

ISSN: 2803-5992

Editore

Presses universitaires de Strasbourg

\section{Edizione cartacea}

Data di pubblicazione: 30 juin 2013

Paginazione: 83-88

ISBN: 978-2-35410-054-4

ISSN: 1968-035X

Notizia bibliografica digitale

Jarmila Očkayová, «Ero sempre una betulla che si aggirava in un boschetto di pioppi», reCHERches

[Online], 10 | 2013, online dal 08 février 2022, consultato il 10 février 2022. URL: http://

journals.openedition.org/cher/11116 ; DOl: https://doi.org/10.4000/cher.11116

\section{(c) (†) (2)}

$\mathrm{Ce}(\mathrm{tte})$ œuvre est mise à disposition selon les termes de la Licence Creative Commons Attribution Pas d'Utilisation Commerciale - Partage dans les Mêmes Conditions 4.0 International. 


\title{
Ero sempre una betulla che si aggirava in un boschetto di pioppi
}

\author{
JARMILA OČKAYOVÁ \\ Scrittrice
}

$\mathrm{L}$ asciare una patria per adottarne un'altra: è una sola porta d'uscita e, allo stesso tempo, d'ingresso. Ed è una porta girevole. La soglia della doppia appartenenza, ma anche di una possibile doppia assenza. Essere qui e là contemporaneamente, o non essere mai davvero in nessun luogo - inteso come luogo mentale - restare sospesi tra il paese d'origine e quello di arrivo. Per anni io dicevo che vivo come se sostassi metaforicamente in un qualche paese delle Alpi austriache, a metà strada tra la Slovacchia e l'Italia, con un piede nel Danubio e l'altro nel Po. Ci vuole un gigante, per reggere una cosa del genere - e infatti la doppia appartenenza può essere un'avventura grandiosa, ricca di potenzialità, come può diventare una cosa spaventevole, fonte di lunghi smarrimenti.

Che sia anzitutto una ricchezza, un fascio di possibilità avuto in dono dal destino, io lo credo soprattutto grazie alla scrittura, che in questo caso offre un'ibridazione feconda: si scrive in una sola lingua, ma nella "penna» agiscono tutte e due, la madrelingua e la lingua adottata, con i loro variegati retroscena culturali, sociali e storici, con le loro simbologie e abitudini cognitive, con i loro anfratti psicologici e retaggi dell'inconscio, con la loro realtà e il loro immaginario. Una doppia identità che si forma a poco a poco e che può essere vissuta, e raccontata, come una scissione, dolorosa - lasciare il proprio paese è una specie di strappo biologico, una ferita che non si rimargina mai completamente, resta aperta sotto i nuovi «innesti»oppure può diventare un raddoppiamento di ogni cosa. Un intreccio, una 
stratificazione, un deposito di intuizioni preziose. Una doppia apertura, nella vita, nella scrittura, una duplice esplorazione della parola e di ciò di cui la parola è portatrice e che il racconto evoca o svela. Per dirla con il linguaggio musicale, è come una polifonia, o una sinfonia, la Sonata di Kreutzer di Beethoveen, dove si rincorrono e dialogano tra di loro un pianoforte e un violino. Per dirla con un'immagine, è come se lo scrittore bilingue mettesse l'uno di fronte all'altro due specchi: gli specchi si riflettono a vicenda, in immagini via via più piccole, dove il termine "piccolo» equivale all'essenza, sfrondata di aspetti secondari. Un confronto tra due mondi che è anche e soprattutto ricerca e tensione verso quel qualcosa di essenziale, essenziale e perciò universale, comune a tutti. Fino alle immagini ultime, negli specchi puntini quasi indecifrabili, nuclei che hanno a che fare con le ragioni più profonde della nostra esistenza. E con il suo mistero.

L'attenzione ai quesiti esistenziali: credo sia questo il primo risultato per chi vive l'esperienza della doppia appartenenza e che poi riecheggia costantemente nella scrittura creativa. Brodskij diceva che l'esilio per uno scrittore è anzitutto una grande lezione di umiltà. $\mathrm{Ci}$ fa sentire come il proverbiale "ago nel pagliaio»: smarriti, insignificanti, affamati di riconoscimento di quella nostra duplice identità, e con i nervi dell'inconscio sempre scoperti e perciò sensibilissimi, attentissimi al fluire della vita, ansiosi di confrontarci non tanto con altri uomini «di penna», quanto con la vita stessa, con la moltitudine umana che ci circonda, con il consorzio umano che ci ospita. "Non l'ambizione guida lo scrittore in esilio», diceva Brodskij, «ma la bramosía di vita». Così, io credo, la letteratura stessa diventa uno strumento di vita, un mezzo di comunicazione, e la vita invece si fa espressione, si fa letteratura, stile, cifra narrativa.

$\mathrm{Ma}$, appunto, considerata quell'ansia di riconoscimento, si corre il rischio di uno smarrimento, il rischio di trasformare quella doppia identità in un minestrone indigesto. Io lo definisco «fregatura platonica». Platone diceva: «quando un uomo, con la parte migliore del suo occhio, la pupilla, guarda la parte migliore dell'occhio dell'altro, vede se stesso». E la gente vuole proprio questo: la possibilità di specchiarsi nello sguardo di chi ha di fronte, ritrovare nell'altro la conferma del proprio mondo e modo di essere. Cerca la propria immagine riflessa, non il diverso. Esattamente come i paesi/luoghi geografici, l'accettazione dello straniero e il confronto con lui hanno i loro confini e spesso sono confini armati, una specie di cortina di ferro mentale. Usando un gioco di parole: «il diverso è benvenuto se è un diversivo», una specie di intervallo esotico o edonico o curioso o utilitaristico tra le cose e 
le sicurezze di sempre. Lo spaziare della mente è per lo più questo: «diverso allargato al divers-iv-o». Negarcelo sarebbe inutile quanto fuorviante, io credo. Credo che l'Italia viva ancora in quello stadio che Umberto Eco già parecchi anni fa definiva «lo shock della differenza». In teoria accogliente e tollerante, in pratica - troppo spesso - ancora piena di pregiudizi, più o meno consapevoli o più o meno inconsci, se non addirittura xenofoba. Amorevole con gli stranieri come la matrigna della fiaba con Cenerentola. Generosa nel concedere la propria lingua e la propria cultura, purché appunto le ritrovi rispecchiate nelle pupille ammirate e acritiche dello straniero.

Intendiamoci: lo specchio platonico dello sguardo va bene, va benissimo, rappresenta appunto quel riconoscimento della comune umanità, della nostra essenza. Purché da specchio platonico la conoscenza dell'altro non diventi la caverna platonica, quella in cui gli uomini sono legati e guardano le ombre proiettate sulle pareti della caverna, anziché le persone in carne ed ossa che passano alle loro spalle e restano non viste. Per conoscere davvero l'altro è necessario attraversare lo specchio, come fa Alice di Lewis Carroll. Anche il suo paese delle meraviglie non è pieno solo di meraviglie, ma anche di insidie, di paure, di incognite; ma bisogna correre il rischio, se non vogliamo conoscere dell'altro solo i cliché, gli stereotipi, i luoghi comuni, i giudizi astratti trasformati in pre-giudizi e agìti in mille e mille minuscole barbarie quotidiane ancora prima che in eclatanti fatti di cronaca.

Ovviamente, in questa mia riflessione vale il principio di reciprocità, anche nella sua accezione negativa: parlo dello straniero, della percezione dello straniero, e per chi arriva da fuori lo straniero è l'italiano.

Per molti anni ho creduto che non ci fosse poi tanta differenza tra la Slovacchia e l'Italia. In fondo, sono due paesi europei, eredi, insieme, della cultura greco-romana da un lato e delle tradizioni giudaico-cristiane dall'altro. Le differenze, mi dicevo, riguardano la storia, le tradizioni locali, parzialmente le abitudini cognitive. Tolte le «sovrastrutture» il nucleo, quell'essenziale dei due specchi, i valori di base, sono gli stessi. Gli stessi i sentimenti e le pulsioni vitali, il senso della felicità e quello della tragedia, il modo di vivere la gioia e il dolore. Anche la lingua la vedevo come uno strumento diverso, come può essere diverso un indumento che si adatta al clima - in un paese freddo si porta il berretto di lana, in un paese assolato il cappello di paglia, ma la testa sotto non è né berretto né cappello, è un'altra cosa.

Fino ad ora ho parlato sempre al passato: mi sembrava, pensavo, credevo... Tra quel passato e oggi c'è una specie di scacco esistenziale, una 
rottura. Luigi Meneghello ha coniato una parola intrigante, che ora viene utilizzata per definire la doppia appartenenza: «dispatrio».

Dis-patrio. Con quel prefisso, «dis», che in tante parole italiane rovescia il senso positivo della parola a cui si prefigge: il simile diventa dissimile, l'armonia disarmonia, il conoscere disconoscere, l'avventura disavventura, il senso dissenso, la parità disparità, e via dicendo. Ecco: dopo tre quinti della mia vita vissuti in Italia, mi sono resa conto che la mia vita è piena di quei prefissi «dis». Che sono diversa, profondamente diversa, e che avevo sempre tenuto quella diversità a guinzaglio, per adattarmi alle situazioni e alle persone. E che quindi, da persona, ma soprattutto da scrittrice, in termini di identità camminavo su un crinale, sottilissimo, tra due versanti: integrazione e disintegrazione. E che l'integrazione e la disintegrazione erano due fianchi della stessa montagna, e non sembrava esserci una terza possibilità.

Il rapporto con il paese d'origine è più un rapporto temporale che spaziale: più che con la lontananza geografica ha a che fare con la distanza nel tempo. È un rapporto tra il presente e il passato, tra ciò che siamo ora $\mathrm{e}$ ciò che eravamo prima in quel dato luogo e che non siamo più o siamo solo molto parzialmente. E tuttavia le nostre radici sono forti, segnate dal nostro codice genetico: abbiamo ereditato ben più della struttura corporea e del colore degli occhi e dei capelli e il nostro primo alfabeto delle esperienze di vita e relativi affetti ed emozioni è indelebile.

Il rapporto con il paese d'adozione invece è un rapporto con un presente senza passato, o passato parziale, senza radici. O meglio: con le radici che vengono da altrove, radici vaganti, che gli altri attorno a noi notano sempre e non dimenticano mai, le tengono presenti ben più di noi e le guardano talvolta con simpatia, ma assai più di frequente con una aprioristica diffidenza. Per dirla con una metafora: «io ero sempre una betulla che si aggirava in un boschetto di pioppi». Tra file regolari e simmetriche di pioppi, dove ero sempre un po' fuori posto. Benvenuta o meno, ma sempre in qualche modo precaria, nella percezione dei miei interlocutori. Imprevedibile e misteriosa, nel migliore dei casi, interpretata e reinventata in quelli peggiori. In una sola parola: straniera. Senza una seconda patria, anche se la nuova non-patria ha inciso sulla mia identità, tracce marcate quanto quelle della mia identità originaria: restando alla metafora dell'albero, se la Slovacchia rappresenta le radici, le esperienze italiane sono la mia chioma, vari rami e ramoscelli dell'albero.

E poi c'è il tronco, che sono io, la mia identità profonda. Complessa e composita, come i cerchi che vediamo nella sezione trasversale del tronco, 
e fatta di qualcosa che comprende e oltrepassa le due estremità dell'albero. La mia identità più profonda è fatta della mia specifica umanità. Todorov, a proposito della doppia appartenenza, parlava della «transculturazione»: un terzo codice culturale. Io, sommando le mie esperienze di vita e quelle letterarie, di letture e di scrittura, vorrei parlare di un quarto codice. E mi piacerebbe definirlo il codice del cuore. Il cuore umano, semplicemente. Inteso come l'aspirazione alla pienezza della vita. E penso, in proposito, alle parole di Paul Valéry. Pascal diceva che «il cuore conosce ragioni che la ragione ignora» e Valéry lo correggeva dicendo: «quelle del cuore non sono ragioni, bensì forze». Il mio codice di persona e di scrittrice è questo: la mia e altrui umanità, umanità spesso offesa e umiliata, talvolta letteralmente massacrata, eppure con una dignità e un calore rimasti intatti.

Con il mio quarto codice, con la mia unicità e l'attenzione al diverso, con la mia tensione verso l'universale, io mi sento e mi definisco anzitutto «essere umano». Mi sento assolutamente cosmopolita, cittadina del mondo, e questo a prescindere dal fatto che io mi muova, viaggi, o stia ferma in un solo luogo. Cosmopolita, e un po' anche "cosmo-dina»: parola inventata per definire il mio sentirmi «citta-dina del cosmo», dell'universo intero, per la mia spinta verso il trascendentale.

Credo che delle forze del cuore questo mondo, quanto mai lacerato e traballante, abbia un gran bisogno. Ma sono anche convinta che farlo accettare, il codice del cuore, non sia affatto semplice, né indolore, specie se lo «straniero» tira fuori parole italiane, diventate tremendamente obsolete, come «equità», «verità», «giustizia». Né semplice né indolore, anzi, sempre più spesso mi sembra del tutto impossibile, in questa Italia ancora strapiena di schieramenti costruiti attorno a polemiche premeditate, strapiena di astrazioni lontane dalla vita e di faziosità, ancorata sempre alla concezione scolastica dell'esistenza, che funziona per divisioni in squadre e tifoserie e dove le credenziali richieste, per essere accettati, come fossero titoli di merito, sono quelle di una precisa appartenenza (che si voglia sociale e politica, più che culturale o antropologica, e che spesso presenta caratteristiche da clan). Ed è come chiedere ad un albero di reggersi non sulle radici o sul tronco, ma su un qualche ramo secondario.

A questo punto della mia vita io mi sento come l'angelo di Walter Benjamin, in quel saggio in cui descrive un quadro di Klee, Angelus Novus ${ }^{1}-$

1 W. Benjamin, Angelus Novus. Saggi e frammenti, a c. di R. Solmi, con un saggio di F. Desideri, Torino, Einaudi, 2006. 
e mi sembra una similitudine applicabile anche in generale a questo paese sempre più multietnico e multiculturale e così poco preparato ad esserlo.

"Quell'angelo» - dice Benjamin - «è in procinto di allontanarsi da qualcosa, su cui fissa lo sguardo». Quel qualcosa è la storia, il passato, frantumato. L'angelo vorrebbe trattenersi, riconnettere i frantumi, ma dal paradiso soffia una bufera che gli scompiglia le ali, lo immobilizza, e al contempo lo spinge verso il futuro, futuro a cui lui, rivolto ancora verso il passato, gira le spalle.

Io sto guardando ben due passati: quello slovacco e quello italiano. Due storie mie, individuali, ma tutte e due intrecciate (e pesantemente condizionate) alla storia con la $\mathrm{S}$ maiuscola. Con una pluri-decennale cittadinanza italiana eppure, paradossalmente, con una vita da apolide, stringo tra le mani il mio kit di ricordi, di sentimenti e di idee, di conquiste e di sconfitte, di simboli e di gesti, mentre il tempo mi spinge verso un futuro che non vedo e continuo a chiedermi se lì, nel futuro - mio, nostro - ci sia ancora spazio sufficiente per la forza salvifica della parola e, soprattutto, per la forza del cuore umano.

E non so rispondermi. 